in vivo $34: 2485-2490(2020)$

doi:10.21873/invivo.12064

\title{
Role of Leptin in Neoplastic and Biliary Tree Disease
}

\author{
PAOLO IZZO $^{1}$, SARA IZZO $^{2}$, PIERFRANCESCO DI CELLO ${ }^{3}$, GABRIELE D'AMATA $^{4}$, \\ MAURIZIO CARDI ${ }^{1}$, ANDREA POLISTENA ${ }^{1}$, DANIELA MESSINEO ${ }^{5}$ and LUCIANO IZZO ${ }^{1}$ \\ ${ }^{1}$ Department of Surgery "Pietro Valdoni”, Policlinico Umberto I, Università degli studi “La Sapienza”, Rome, Italy; \\ ${ }^{2}$ Unit of Colorectal Surgery, Department of Medical, Surgical, Neurologic, Metabolic and Ageing Sciences, \\ University of Campania "Luigi Vanvitelli", Naples, Italy; \\ ${ }^{3}$ UOC Chirurgia Generale Frosinone-Alatri presso ASL Frosinone, Frosinone, Italy; \\ ${ }^{4}$ ASL Roma 5 UOC Chirurgia Generale Ospedale di Colleferro, Rome, Italy; \\ ${ }^{5}$ Department of Radiological, Oncological and Anatomo-Pathological Sciences, \\ Università degli Studi “La Sapienza”, Rome, Italy
}

\begin{abstract}
Background/Aim: Leptin is a small hormone of protein nature, it is strongly involved in the regulation of lipid metabolism and its functioning mechanism is not yet well known or whether or not it is actually secreted by cholangiocytes, nor if the biliary tree expresses its receptors. In the past, various studies have tried to correlate leptin levels with certain neoplasms. The aim of this study was to demonstrate that serum leptin values can become a new sensitive and specific serum marker for cholangiocarcinoma. Materials and Methods: Seventy-two patients with gallbladder stones, hepatolithiasis with benign biliary stenosis, cholangiocarcinoma, and a group of patients without hepatobiliary diseases were enrolled in the study. In all cases blood and bile samples were collected for evaluation of leptin levels and liver biopsies were performed to confirm diagnosis. In all patients, both ultrasound and cholangio-magnetic resonance imaging (MRI) were performed to complete the diagnostic procedure. Results: Twenty-two patients were affected by cholangiocarcinoma, 50 by benign biliary disease $(35$ cholelithiasis and 6 hepatolithiasis and 9 by inflammatory biliary stenosis). The mean values of serum leptin in patients with cholangiocarcinoma were $19.28 \pm 8.76 \mathrm{ng} / \mathrm{ml}$, significantly higher than those observed in non-neoplastic biliary diseases. Conclusion: Serum leptin levels might be a useful marker to differentiate patients with cholangiocarcinoma from those with biliary lithiasis and inflammatory stenosis.
\end{abstract}

This article is freely accessible online.

Correspondence to: Luciano Izzo, Department of Surgery "Pietro Valdoni", Università degli Studi di Roma "La Sapienza", Viale del Policlinico, 15500161 Rome, Italy. Tel: +39 0649970322, Fax: +39 0649970322,e-mail: luciano.izzo@uniroma1.it

Key Words: Biliary neoplasm, markers, serum, leptin, cholelithiasis.
Cholangiocarcinoma is a rare tumour (representing less than $2 \%$ of all malignancies) (1) and is ranked, in order of frequency, as the second primary liver tumour after hepatocarcinoma. It occurs mainly in the seventh decade of life (range $=50-70$ years) with a slight predisposition in the male sex $(2,3)$. A racial predisposition is not certain. It is generally classified according to the anatomical site of onset in intrahepatic (IC) and extrahepatic (HC) (2-4). Three types of intrahepatic cholangiocarcinomas have been described according to the type of macroscopic growth: mass forming (MF), periductal infiltrating (PI), intraductal growing (IG) (2). $\mathrm{HC}$ is classified internationally according to the Bismuth and Corlette scheme. These tumours may have a greater intrahepatic or extrahepatic development (4). Some authors have correlated the incidence of cholangiocarcinoma with cholelithiasis (5): biliary stasis with over infection and chronic inflammation are decisive elements in the pathogenesis of the neoplasms and these conditions can be supported by various diseases $(1,6)$. The biomolecular basis of cholangiocarcinoma development (1-4) has been identified in several experimental studies, highlighting pathogenic mechanisms that have remained obscure for many years. In particular, the association between chronic cholestasis and phenotypic cholangiocyte mutation has been clarified (5). Biliary infestations with Opistorchis viverrini and Clonorchis sinensis, primary sclerosing cholangitis (PSC), biliary stones, Caroli's disease and congenital cystic dilations of the choledochus increase the risk of developing cholangiocarcinoma. However, there are two basic conditions associated with the development of cholangiocarcinoma and they are the chronic inflammation of the biliary epithelium and its subsequent polytrauma up to the obstruction of the Hering's canal $(1,7,8)$. Various cytokines released from biliocytes during chronic inflammation have been shown to be responsible for the process of carcinogenic initiation $(1,7,8)$. Recent studies document how cholangitis produce cytokines with paracrine action such as: interleukin-6 
(IL-6), transforming growth factor- $\beta$ (TGF- $\beta$ ), IL- 8 , tumor necrosis factor- $\alpha$ (TNF- $\alpha$ ) and platelet-derived growth factor (PDGF) B chain $(9,10)$. Cytokines also induce the production of iNOS (inducible nitric oxide synthase) in cholangiocytes (9), an event very much related to malignant transformation. Modern studies suggest that cholangiocarcinoma may originate from hepatic stem cells (11). This hypothesis is strongly supported by experiments performed on mixed forms of hepato-cholangiocarcinoma, a type of tumour with intermediate characteristics: the explanation for this phenomenon is that this neoplasm would derive from progenitor cells with mixed differentiation potential, hepatocyte and cholangiocyte (11). The only therapy for cholangiocarcinoma is complete surgical resection leading to histologically-negative resection margins. Only a few patients are operable, so patients should be carefully evaluated for resection. For example, inflammation or a stent placement infection often makes evaluation difficult (2). The type and extent of surgery depends on the location of the tumour within the liver or biliary tract. In cases of intrahepatic cholangiocarcinoma, it has been established that resection gives better results than other types of intervention (3). After resection, 5-year survival ranges from $8 \%$ to $47 \%$ with the highest survival in patients with negative resection margins (1). Leptin (from the Greek root leptos which means lean) is a small hormone of a protein nature, it is strongly involved in the regulation of lipid metabolism and energy consumption. The biochemical structure of leptin looks like the cytokines with long-helix chains, this group of substances is a sort of bridge of communication between the neuroendocrine system and the immune system. Leptin is carried to the target organs by the blood stream. Its receptors are located mainly within the brain, namely in the hypothalamus. Leptin reduces the sense of hunger (anorectic effects) and increases energy expenditure, helping to reduce body weight and fat mass. Leptin is also involved in the regulation of numerous biological functions: 1) it regulates thyroid activity; 2) it facilitates haematopoiesis; 3) it regulates the immune system (Leptin strengthens the immune system until it triggers real autoimmune diseases); 4) it regulates the reproductive system (it helps the secretion of gonadotropins; it is also produced by the placenta), and 5) it regulates bone formation. Our study aimed to demonstrate that serum leptin values can become a new sensitive and specific serum marker for cholangiocarcinoma. We focused our study on leptin, since we do not currently know whether it is secreted by cholangiocytes, nor whether the biliary tree expresses its receptors. However, considering the very recent evidence and implications in the genesis of some neoplasms such as those of the breast, this Leptin (adipoquin) was chosen as a possible marker for our study. In addition, the determination of the bile salts content of all the samples collected will be carried out by means of a specific colorimetric method and subsequent evaluation of the parameters with the indices of sensitivity and specificity.

\section{Materials and Methods}

In the period between January 2006-June 2009 and January 2015-September 2015, 72 patients with gallbladder stones, hepatolithiasis with benign biliary stenosis, cholangiocarcinoma, and a group of patients without hepato-biliary diseases were selected at the 1st Department of Surgery, Policlinico Umberto I in Rome. All enrolled patients gave their consent to the study and use of data for research. A total of 72 findings in patients with inflammatory and/or tumor disease divided as follows are registered. A total of 50 samples of inflammatory liver material, 22 samples of liver material in cancer (cholangiocarcinoma). In all 72 cases serum and blood samples were taken as a protocol as well as liver samples and sent to the Human Anatomy Centre of the University of Rome La Sapienza, which collaborated in our study. In all 72 patients, both ultrasound and cholangio-MRI were performed to complete the diagnostic procedure (Figure 1). The total of serum and bile samples were collected by blood sampling and during esophagogastroduodenoscopy or CPRE after administration of magnesium sulphate $30 \mathrm{~min}$ before the examination and subsequent collection by suction with $10 \mathrm{Fr}$ cannula, while the histological collection was performed with liver biopsy. The collected serum and bile samples should be of the same quantity, quality and homogeneity. A fasting blood sample $(30 \mathrm{ml})$ was collected and divided into aliquots and frozen at -70 degrees. The samples were de-identified by a unique study number. The serum obtained from each of the patients was centrifuged at $4,000 \mathrm{rpm}$ at $4^{\circ} \mathrm{C}$ and then divided into 4 aliquots, each of $500 \mu \mathrm{l}$, which were immediately placed in a freezer at $-80^{\circ} \mathrm{C}$. Leptin assay on serum samples belonging to 63 healthy patients was performed on the whole serum, while tests on pathological patients were performed with a 1:2 dilution of the serum, to avoid the phenomenon of Hook, i.e. an underestimation of the results due to the presence of excess antigen.

An ELISA kit (Roche Diagnostics, Leptin) was used for the enzyme immunoassay. We used a radioimmunological method to measure basal levels of leptin (12). The serum samples, with appropriate dilutions, were delivered in a microwells plate previously coated with a monoclonal leptin capture antibody, after appropriate incubation at $37^{\circ} \mathrm{C}$. Subsequently, repeated washes were carried out with the aim of removing the "unbound". The colour change was measured spectrophotometrically at a wavelength of $450 \mathrm{~nm} \pm 2 \mathrm{~nm}$. The concentration of leptin in the samples was then determined by comparing the optical density (O.D) of the samples, on the standard curve. The collected liver fragments $(0.5 \mathrm{~mm})$ were fixed in $10 \%$ formalin for 2-4 $\mathrm{h}$ and soaked at low tempera in fused paraffin at $55-75^{\circ} \mathrm{C}$, while sections of 3-4 micrometers will be placed in hematoxylin-eosin. For immunohistochemistry, they were placed on slides treated with $0.1 \%$ poly-Lysine. After deparaffinization, endogenous peroxydasic activity will be blocked by a 30 -min incubation in $2.5 \%$ peroxydasic metabolic 

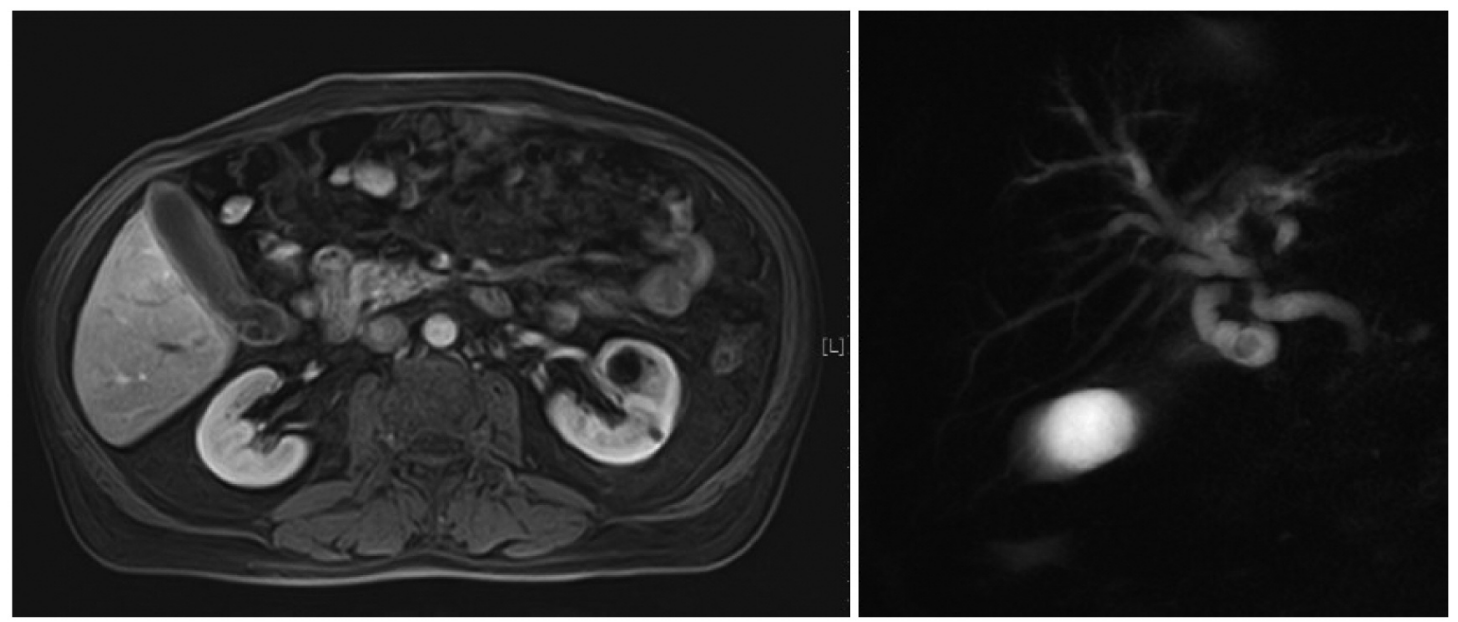

Figure 1. Cholangio-MR showing thickened and edematous walls of the gallbladder and the presence of lithiasic formations, one of which is positioned at neck level and biliary mud.

hydrogen. Sections will be hydrated in graduated alcohol and placed in phosfatasic saline solution, first applying primary antibodies. Sections with a thickness of 3 microns from fabric blocks used as representative tests were cut, paraffined with xylene, rinsed and rehydrated with distilled water through alcohol. The whole was enriched by slides in autoclave to 10 $\mathrm{nM}$ sodium citrate buffer ( $\mathrm{pH}$ 6.0) for $30 \mathrm{~min}$. Endogenous peroxidase was extinguished by incubation in $0.3 \%$ hydrogen peroxide-methanol buffer for 30 minutes. The slides were then incubated overnight at $4^{\circ} \mathrm{C}$ in humidified chambers with primary polyclonal rabbit Antileptin antibodies at a dilution of 1:100. Tumour expression levels have been marked as positive or negative depending on the presence or absence of staining both on the tumour and on the surrounding tissue, in view of the fact that Leptin is predominantly located in the cytoplasmic portions of both tumour and non-cancer liver tissue. Finally, the specimens will be reinserted into the phosphatase salt solution for $5 \mathrm{~min}$, incubated for $10 \mathrm{~min}$ at room temperature with secondary antibodies $(12,13)$. The results were after all statistically evaluated with three populations, the first with cholangiocarcinoma patients, the second with the group of inflammatory diseases and the third with a population similar in age and sex enrolled in healthy patients. Data collected from laboratory tests and processed with Fisher's F test were measured, which allowed to verify the homogeneity of the variances, obtaining a $p<0.05$, index of non-homogeneity between samples.

\section{Results}

A total of 72 patients were evaluated for serum and bile leptin levels and biopsied. In this group of patients 22 were affected by cholangiocarcinoma, 50 by benign biliary disease
(35 cholelithiasis and 6 hepatolithiasis and 9 by inflammatory biliary stenosis) (Figure 2). An evaluation of the serum dose diagnostic value of leptin was carried out. The mean serum leptin concentration was $5.0 \mathrm{ng} / \mathrm{ml}$ (range $=1.4-26.6 \mathrm{ng} / \mathrm{ml}$ ). The mean values of serum leptin in patients with cholangiocarcinoma were $19.28 \pm 8.76 \mathrm{ng} / \mathrm{ml}$, and $5.20 \pm 4.60 \mathrm{ng} / \mathrm{ml}$ in patients with benign disease, and $2.37 \pm 1.19 \mathrm{ng} / \mathrm{ml}$ in healthy controls $(p=0.01)$ (Figure 3).

Patients with cholangiocarcinoma showed significantly higher values of leptin especially patients with lithiasic or inflammatory biliary pathology and the healthy patient group. Patients with cholangiocarcinoma had statistically significant higher values compared to patients with lithiasis $(p=0.001)$, to patients with hepatolithiasis and inflammatory stenosis $(p=0.01)$, and to healthy controls $(p=0.001)$. Patients with lithiasic disease or benign stenosis presented significantly higher values compared to healthy controls $(p=0.01)$. At the second check-up or test of the 22 patients, 19 were diagnosed positive for the disease while 3 were false-positives. On the contrary, out of 50 negative patients 48 were confirmed negative and 2 were positive. Sensitivity had a value of 0.904 , while specificity was 0.906 . Since the sample is numerically small, it was appropriate to study the confidence interval used to quantify the precision of the estimate obtained regarding the values of sensitivity and specificity. IC95\% value of sensitivity shows values ranging from 0.803 to 0.984 , while IC $95 \%$ of specificity ranges from 0.807 to 0.973 . The positive predictive value (VPP) indicating the probability that a patient with a positive test is sick is 0.8636 , while the negative predictive value (NPV) indicating the probability that a patient with a negative test is healthy is 0.96 . 


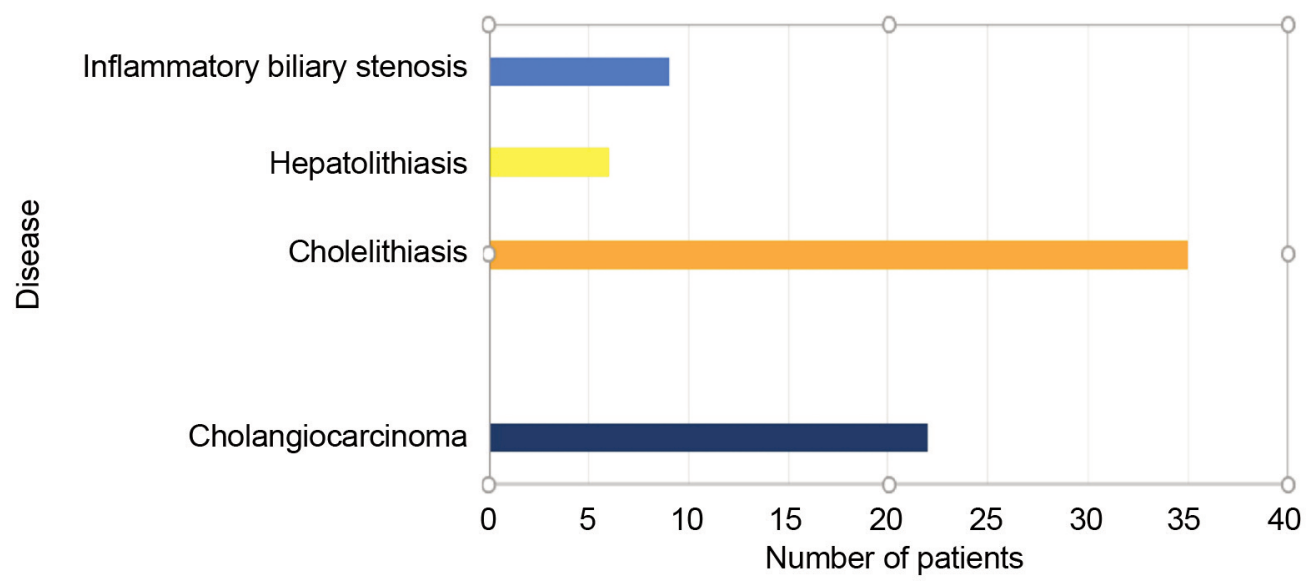

Figure 2. Population of patients included in the study.

\section{Discussion}

Late clinical onset and non-specific signs and symptoms, like those of many other non-neoplastic diseases of the liver and biliary tract, are some of the elements that contribute to the difficulty in the diagnosis and early recognition of cholangiocarcinoma. Even now there are no specific tumour markers for cholangiocarcinoma $(13,14)$. The most used serum markers are carbohydrate antigen (CA19.9) and carcino-embryonic antigen (CEA). The sensitivity and specificity of these markers is low, and they cannot provide a certain diagnosis of cholangiocarcinoma, they can have high values even for benign disease $(15,16)$.

CA19-9 is the most used marker for cholangiocarcinoma. This cancer-associated antigen is penalized in sensitivity and specificity, both in terms of the differential diagnosis between benign and malignant bilio-pancreatic pathologies, and in the diagnosis of this specific tumor. The use of this marker in the diagnosis of cholangiocarcinoma becomes even less useful and less sensitive in the presence of jaundice and cholangitis. Our study aimed to demonstrate that serum leptin values can become a new sensitive and specific serum marker for cholangiocarcinoma $(17,18)$. Leptin regulates body weight by reporting information to the brain regarding the availability of energy stored as fat. This negative feedback cycle is interrupted in more obese individuals and results in resistance to leptin. Several studies report that leptin is a risk factor for carcinogenesis in various sites of organs, including the liver, in fact leptin can stimulate cell proliferation in various types of cancer cells such as those undergoing hepatocarcinoma. Concentrating in the liver, leptin is a potent profibrogenic cytokine. Leptin shows, instead, an aberrant expression in the tissue involved in the neoplasm suggesting that bile epithelial cells assume a different phenotype during carcinogenesis (17, 18). The presence of this aberrant expression at tissue level is

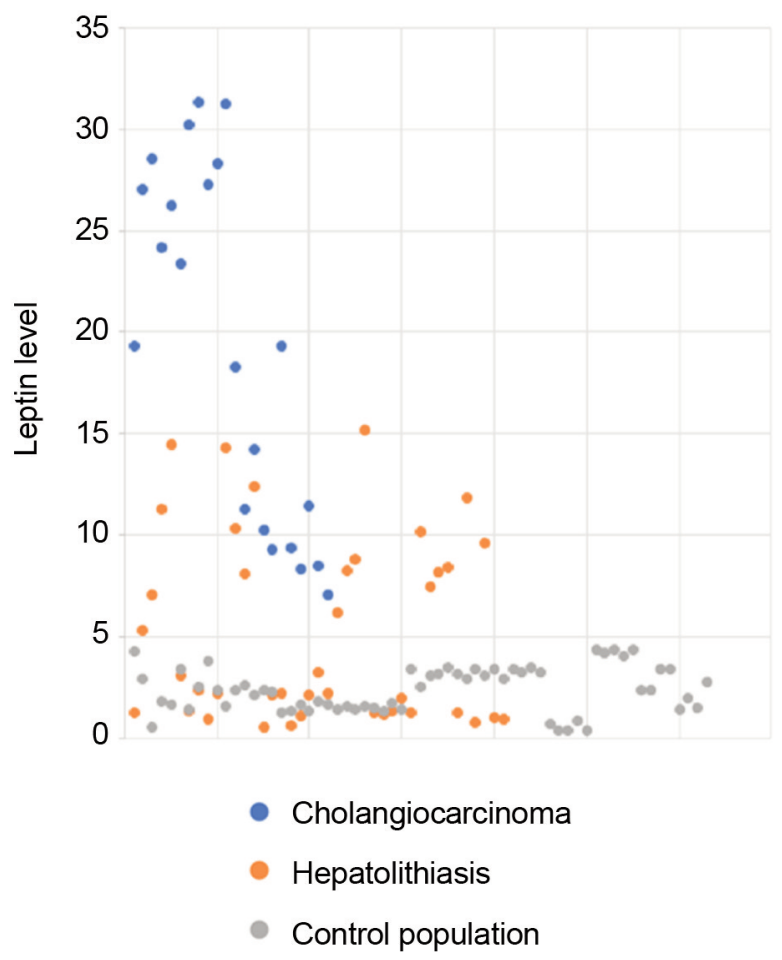

Figure 3. Distribution of leptin values among the examined population.

associated with an increase of leptin in serum, in fact the secretion by neoplastic cells is secondary to mechanical occlusion of the bile ducts, loss of polarity of bile cells and/or early invasion of blood vessels.

In addition, increased expression of leptin is associated with an increase in micro-vascular intratumoral density (17). Our study is one of the first to investigate the diagnostic value of serum leptin dosage in groups of patients with 
cholangiocarcinoma, in patients with gallbladder disease, in patients with hepatolithiasis and inflammatory stenosis (18). We believe that in the future it will be important to also deepen this value in patients with extrahepatic cholangiocarcinoma. In fact, even in recent articles there is no evaluation of this parameter (19). This test can be a useful clinical tool in the differential diagnosis of patients with neoplastic and inflammatory diseases where imaging methods and other dosages do not give reliable results (18). Although the size of the sample does not allow for conclusive considerations to be made, we showed preliminary evidence that serum values of leptin are related to the disease. We did not correlate the form or degree of neoplasm or the tumor stage with the values of leptin because this study is at an early stage and this will be the aim of further research with a higher number of patients.

\section{Conclusion}

Our study, although low in number of included patients, allows us to outline the role of serum leptin dosage as a new serum marker in cholangiocarcinoma. Serum leptin dosage has made it possible to differentiate patients with cholangiocarcinoma from those with biliary lithiasis, with inflammatory stenosis.

\section{Conflicts of Interest}

The Authors have no conflicts of interest to declare in regard to this study.

\section{Authors' Contributions}

Paolo Izzo mainly acquired and analyzed the data and wrote the draft and designed the research. Sara Izzo interpreted the data, and contributed to writing and editing the article. Pierfrancesco Di Cello, participated in the data acquisition. Gabriele D'Amata, participated in data acquisition. Massimo Codacci Pisanelli, participated in data acquisition. Andrea Polistena participated in data acquisition and contributed to editing the article. Daniela Messineo participated in data acquisition and contributed to editing the article. Luciano Izzo supervised the research design, interpretation of the data and contributed to editing the article. All Authors participated in critical revision of the article for important intellectual content.

\section{Acknowledgements}

The Authors would like to thank the Colleagues and Technicians of the Department of the Human Anatomy Centre of the University of Rome La Sapienza laboratories for their kind cooperation as they made this study possible.

\section{References}

1 Lazaridis $\mathrm{KN}$ and Gores GJ: Cholangiocarcinoma. Gastroenterology 128: 1655-1667, 2005. PMID: 15887157. DOI: $10.1053 /$ j.gastro.2005.03.040
2 Valle JW, Borbath I, Khan SA, Huguet F, Gruenberger T and Arnold D: ESMO Guidelines Committee Biliary cancer: ESMO Clinical Practice Guidelines for diagnosis, treatment, and followup. Ann Oncol 27(5): v28-v37, 2016. PMID: 27664259. DOI: 10.1093/annonc/mdw324

3 Berthiaume EP and Wands J: The molecular pathogenesis of cholangiocarcinoma. Semin Liver Dis 24: 127-137, 2004. PMID: 15192786. DOI: $10.1055 / \mathrm{s}-2004-828890$

4 Foja S, Goldberg M, Schagdarsurengin U, Dammann R, Tannapfel A and Ballhausen WG: Promoter methylation and loss of coding exons of the fragile histidine triad (FHIT) gene in intrahepatic cholangiocarcinomas. Liver Int 25: 1202-1208, 2005. PMID: 16343073. DOI: 10.1111/j.1478-3231.2005.01174.x

5 Shaib Y and El-Serag HB: The epidemiology of cholangiocarcinoma. Semin Liver Dis 24(2): 115-125, 2004. PMID: 15192785. DOI: $10.1055 / \mathrm{s}-2004-828889$

6 Marzioni M, Fava G, Alvaro D, Alpini G and Benedetti A: Control of cholangiocyte adaptive responses by visceral hormones and neuropeptides. Clin Rev Allergy Immunol 36(1): 13-22, 2009. PMID: 18548352. DOI: 10.1007/s12016-008-8090-1

7 Gores GJ: Cholangiocarcinoma: current concepts and insights. Hepatology 37(5): 961-969, 2003. PMID: 12717374. DOI:10.1053/ jhep.2003.50200

8 Pearngam $\mathrm{P}$, Kumkate $\mathrm{S}$, Okada $\mathrm{S}$ and Janvilisri $\mathrm{T}$ : Andrographolide inhibits cholangiocarcinoma cell migration by down-regulation of claudin-1 via the p-38 signaling pathway. Front Pharmacol 10: 827, 2019. PMID: 31404237. DOI: 10.3389/fphar.2019.00827

9 Taniai M, Grambihler A, Higuchi H, Werneburg N, Bronk SF, Farrugia DJ, Kaufmann SH and Gores GJ: Mcl-1 mediates tumor necrosis factor-related apoptosis-inducing ligand resistance in human cholangiocarcinoma cells. Cancer Res May 64(10): $3517-$ 3524, 2004. PMID: 15150106. DOI: 10.1158/0008-5472.CAN-032770

10 Huang T, Ding X, Xu G, Chen G, Cao Y, Peng C, Shen S, Lv Y, Wang L and Zou X: CDK7 inhibitor THZ1 inhibits MCL1 synthesis and drives cholangiocarcinoma apoptosis in combination with BCL2/BCL-XL inhibitor ABT-263. Cell Death Dis 10(8): 602, 2019. PMID: 31399555. DOI: 10.1038/s41419019-1831-7

11 D'Andrea V, Guarino S, Di Matteo FM, Maugeri Saccà M and De Maria R: Cancer stem cells in surgery. G Chir 35(11-12): 257-259, 2014. PMID: 25644725.

12 Owecki M, Nikisch E, Miczke A, Pupek-Musialik D and Sowiński J: Leptin, soluble leptin receptors, free leptin index, and their relationship with insulin resistance and BMI: high normal BMI is the threshold for serum leptin increase in humans. Horm Metab Res 42(8): 585-589, 2010. PMID: 20455195. DOI: $10.1055 / \mathrm{s}-0030-1253422$

13 Carmienke S, Freitag MH, Pischon T, Schlattmann P, Fankhaenel $\mathrm{T}$, Goebel $\mathrm{H}$ and Gensichen J: General and abdominal obesity parameters and their combination in relation to mortality: a systematic review and meta-regression analysis. Eur J Clin Nutr 67(6): 573-585, 2013. PMID: 23511854. DOI: 10.1038/ejen.2013. 61

14 Buechler C, Haberl HM, Fischboeck LR and Aslanidis C: Adipokines in liver cirrhosis. Int J Mol Sci 18(7): 1392, 2017. PMID: 28661458. DOI: 10.3390/ijms18071392

15 Li O, Yi W, Yang P, Guo C and Peng C: Relationship between serum MMP-9 level and prognosis after radical resection for 
Hilar cholangiocarcinoma patients. Acta Cir Bras 34(4): e201900409, 2019. PMID: 31038586. DOI: 10.1590/s0102865020190040000009

16 Okada N, Ishida H, Murata N, Hashimoto D, Seyama Y and Kubota S: Matrix metalloproteinase-2 and -9 in bile as a marker of liver metastasis in colorectal cancer. Biochem Biophys Res Commun 288: 212-216, 2001. PMID: 11594775. DOI: $10.1006 / \mathrm{bbrc} .2001 .5741$

17 Peng C, Sun Z, Li O, Guo C, Yi W, Tan Z and Jiang B: Leptin stimulates the epithelial-mesenchymal transition and proangiogenic capability of cholangiocarcinoma cells through the miR-122/PKM2 axis. Int J Oncol 55(1): 298-308, 2019. PMID: 31115511. DOI: $10.3892 /$ ijo.2019.4807

18 Fava G, Alpini G, Rychlicki C, Saccomanno S, DeMorrow S, Trozzi L, Candelaresi C, Venter J, Di Sario A, Marzioni M, Bearzi I, Glaser S, Alvaro D, Marucci L, Francis H, Svegliati-
Baroni G and Benedetti A: Leptin enhances cholangiocarcinoma cell growth. Cancer Res 68(16): 6752-6761, 2008. PMID: 18701500. DOI: 10.1158/0008-5472.CAN-07-6682

19 Morino K, Seo S, Yoh T, Nishino H, Yamanaka K, Fukumitsu K, Ishii T, Taura K, Okajima H, Kaido T and Uemoto S: The efficacy and limitations of postoperative adjuvant chemotherapy in patients with extrahepatic cholangiocarcinoma. Anticancer Res 39(4): 21552161, 2019. PMID: 30952762. DOI:10.21873/anticanres.13329

Received June 19, 2020

Revised July 7, 2020

Accepted July 8, 2020 